\title{
IDENTIDADES Y VALORES EN TENSIÓN: LOS INICIOS DEL HOCKEY SOBRE CÉSPED EN ARGENTINA
}

\section{IDENTITIES AND VALUES: THE BEGINNING OF FIELD HOCKEY IN ARGENTINA}

\author{
Ángela Marcela Aisenstein ${ }^{1}$ \\ María Andrea Feiguin ${ }^{2}$
}

Resumen

El presente trabajo describe el origen y desarrollo de la práctica del hockey sobre césped en la Argentina en el período comprendido entre los años 1903 y 1930, y su vinculación con la comunidad británica, tomando como fuente uno de sus periódicos: The Standard. De su análisis puede decirse que en dicha etapa el juego estuvo signado por continuidades y cambios en los significados atribuidos, en el tipo de instituciones donde fue jugado, en la modalidad de organización y en los valores que caracterizaron su práctica. Otro aspecto destacable fue el rol asumido por el diario.

Palabras clave: Argentina, hockey sobre césped, inmigración, valores, identidades.

Abstract

This paper describes the beginning and practice development of field hockey in Argentina from 1903 to 1930. This covers the role of the British community taking as a source one of its newspapers, The Standard. From analysis we can say during that period this sport was characterized by continuum and changes of meanings and nature of institutions where it was played, besides types of organization and values typifying its practice. Another highlight was the role by the newspaper.

Keywords: Argentine, field hockey, immigration, values, identities.

Fecha de recepción: 26 de junio de 2012

Fecha de aprobación: 28 de agosto de 2012

1 Dra. en Educación. Magíster en Ciencias Sociales. Licenciada en Educación. Profesora de Educación Física Universidad de San Andrés/Universidad Nacional de Luján. aaisenstein@gmail.com 2 Maestranda en Ciencias Sociales con orientación en Historia, Universidad Nacional de Luján. Licenciada en Educación Física. Profesora de Educación Física. mafeiguin@yahoo.com.ar 


\section{Introducción}

El presente trabajo describe el origen y desarrollo de la práctica del hockey sobre césped en la Argentina en el período comprendido entre los años 1903 y 1930, y su vinculación con la comunidad británica, tomando como fuente uno de sus periódicos: The Standard.

Según Devoto (2003) y Graham Yooll (2007), dentro del conglomerado étnico que conformaba la Argentina desde fines del siglo XIX, cada grupo inmigratorio dejaría una impronta en la cultura de la república. Para el caso de los "ingleses", si bien la cantidad de arribados no fue significativa respecto de otras comunidades, sí lo fue cualitativamente ${ }^{1}$. Dueños de una posición social que los diferenciaba de los llamados "inmigrantes", los "ingleses", como se conocía a todo angloparlante indistintamente fuera inglés, escocés, irlandés o galés, se autoconsideraban y se autodenominaban extranjeros o visitantes. Inspirados en el deseo de "vivir como en su país de origen", conformaron instituciones -escuelas, clubes, iglesias- que les permitieron conservar su cultura y mitigar el sentimiento de desarraigo. Esta concepción hizo que el aporte de los viajeros anglosajones también tuviera una importante influencia en el plano sociocultural; una de las costumbres que llevaron fueron los pasatiempos y deportes, entre ellos el hockey. Dentro de ese cuadro la difusión mundial de los deportes, la organización de escuelas, la fundación de clubes y la Primera Guerra Mundial marcaron puntos de inflexión dentro del proceso de inclusión y difusión del hockey sobre césped en la Argentina. Proceso signado por cambios y continuidades en los significados atribuidos, el tipo de instituciones donde fue jugado, la modalidad de organización y los valores que caracterizaron su práctica durante el período aquí abordado.

Se ha tomado el año 1903 como inicio del período, porque a ese año corresponde el primer artículo en el diario The Standard que hace referencia al hockey, y como hito de cierre el año 1930 por ser el momento en que equipos del interior del país ${ }^{2}$ se incorporaron por pri-

1 Gran Bretaña, especialmente Inglaterra, era una de las referencias políticas, económicas y culturales. Con el ingreso a la Argentina de capitales ingleses organizados en forma de empresas, bancos, aguas corrientes, industria de la alimentación y sobre todo los ferrocarriles, también llegaron su religión, su idioma, sus escuelas y sus deportes.

2 Una parte de la comunidad inglesa se concentró en algunos barrios de la ciudad de Buenos Aires (por ej. Belgrano, Barracas), así como también en algunos suburbios de lo mera vez a las actividades programadas por la A.A.H. (Asociación Argentina de Hockey); la fuente fue el diario The Standard y la técnica de recolección de datos el análisis temático de contenidos ${ }^{3}$.

The Standard era un diario editado en inglés, fundado en 1861 por los hermanos Mulhall. No era el único diario editado en ese idioma; existía otro denominado Herald fundado en 1876 por un descendiente de un miembro de la colonia escocesa de Monte Grande, que lo vendió a un norteamericano en 1877. Puede decirse que frente al crecimiento británico en el país, y dado que muchos de los residentes británicos en Argentina no hablaban ni entendían castellano, The Standard actuó como medio de información tanto de lo que ocurría en el "viejo país" y en Europa, como también de lo que ocurría en Argentina.

The Standard, considerado el diario de los británicos, era la fuente de información y espacio para la convocatoria de jugadores. Además de difundir aquello que ocurría en las distintas esferas, sus páginas constituían una "mesa de debate" en donde editor y entusiastas intercambiaban opiniones y posturas respecto a cómo debía ser concebida la práctica del hockey en Argentina.

que actualmente se conoce como Gran Buenos Aires (Hurlingham, San Isidro, San Martín, Quilmes, Lomas de Zamora). Otra se radicó en ciudades del interior de las provincias de Buenos Aires (ej. Junín), Entre Ríos (ej. Concordia) y Santa Fe (ej. Rosario, Venado Tuerto). Los nombres de los equipos y luego clubes derivarían de esta identificación territorial. 3 El trabajo de archivo se llevó a cabo en la Biblioteca Nacional, sección publicaciones periódicas antiguas, donde se realizó la lectura y toma de fotos de artículos e imágenes relevantes para este trabajo encontrados en cada ejemplar desde 1880 a 1931. La recolección de datos para este estudio histórico descriptivo consistió en la recopilación, selección y análisis de noticias, avisos publicados en las columnas Sports Hockey, Telephonia, Editor's Table del diario The Standard desde 1880 hasta 1931, llegando a contabilizar un total de 18 600 ejemplares. La cantidad de avisos publicados resultó uno de los indicadores de la actividad alrededor de este deporte y del apoyo del diario al hockey. Desde 1903 las publicaciones aumentaron año a año; llegaron a publicarse nueve avisos en el año 1905. A partir de 1908 y con la excepción de los años de la "Gran Guerra", las notas y noticias no volvieron a disminuir. En el año 1930 se llegaron a publicar 856 notas. Si bien la elección de una sola fuente pudo haber resultado una limitación para el objetivo de conocer el proceso de difusión de este deporte en el país, la lectura minuciosa del diario permitió identificar no solo las discusiones y planteos realizados por los entusiastas del hockey, sino reconocer el papel jugado por el propio diario en el proceso que se pretendía indagar. 
De ahí que puede decirse que el diario cumplía un doble rol: informador y actor en el proceso de difusión y organización del deporte. En su rol de informador era el principal medio de comunicación por el cual los residentes oriundos del "viejo país" se enteraban de todas las actividades que acontecían aquí y en Inglaterra (fueran comerciales, educativas, políticas, sociales o deportivas). También participaba como un actor social relevante en las cuestiones que atañían a la comunidad británica en el país. Se puede observar cómo, con una clara actitud intervencionista, a través de sus notas y noticias apoyó el crecimiento y direccionó el desarrollo del hockey. Quien estuviera interesado en jugar o ver hockey debía leer The Standard para enterarse de novedades o programas del juego:

Es posible que el partido sea postergado por lluvia u otras modificaciones. Los jugadores deberán leer el The Standard para enterarse de los arreglos finales. (The Standard, julio de 1911, p. 5)

Resulta importante señalar que muchos deportes hoy practicados en todo el mundo, se originaron principalmente en Inglaterra y de ahí se extendieron a diferentes países durante la segunda mitad del siglo XIX y la primera mitad del siglo XX. Los deportes anglosajones, entendiendo a estos como al conjunto de pasatiempos ingleses transformados en el marco del proceso de industrialización (Elias y Dunning, 1992), se caracterizaron por la estandarización, racionalización y adopción de medidas que comenzaron a ser integrados en la vida y cultura inglesa, dando inicio a nuevas características de los pasatiempos populares. Estos deportes modernos de raíz occidental -definidos por la presencia o ausencia de un grupo interrelacionado de características formales y estructurales como secularismo, igualdad, especialización, cuantificación, obsesión por los récords, entre otras- se difundieron de una nación a otra por el poder político, económico y cultural de las naciones involucradas. Los viajeros angloparlantes fueron algunos de los que favorecieron las reformas en la educación y en los juegos llevando consigo sus costumbres a todo sitio al que llegaba su bandera (Guttman, 1994; Mandell, 1984).

De esta forma los pasatiempos y deportes jugados en Inglaterra comenzaron a ser adoptados en cada rincón del mundo donde arribaron "ingleses". Ejemplo de ello fue la práctica del fútbol, rugby, tenis, cricket, hockey, remo. La comunidad británica denominaba pastime a las diferentes formas de recreación que implementaban para los momentos de ocio. En ese marco, el térmi- no "deporte" se generalizó como término técnico para designar formas de recreación en las cuales el ejercicio físico desempeñaba un papel fundamental; formas específicas de recrearse que se desarrollaron primero en Inglaterra y luego se extendieron por todo el mundo (Elias y Dunning, 1992, p.185).

\section{La deriva del hockey en las páginas de The Standard}

El análisis de las notas del diario permite señalar que inicialmente la práctica del hockey estuvo centrada en la comunidad británica radicada fundamentalmente en Buenos Aires, y que la ampliación de su práctica fue un proceso irregular en el que pueden identificarse diferentes períodos caracterizados a partir de las similitudes y/o diferencias halladas en torno a tres cuestiones: los valores alrededor de los cuales se organizaba, el grado de institucionalización y los criterios para la formación de los equipos, y el rol del diario en todo ese proceso.

En relación al primer aspecto, la ética de afición, es decir el participar por diversión, como expresión de honor, de generosidad y de placer en el juego mismo, fue por momentos el valor rector de la práctica del hockey. También, la concepción británica del deporte era asociada al rol civilizador y modelador de la conducta como expresión de honor, camaradería y juego limpio (Archetti, 2001). En ese marco, el objetivo de la práctica del hockey era -reafirmando la idea de "viajeros"- reproducir el espíritu inglés a través de sus costumbres y tradiciones.

Por otra parte, el placer por el juego mismo, propio del deporte amateur y de los pasatiempos ingleses, se conjugó con ideas de participación "dirigidas al otro" (Elias y Dunning, 1986) que dejaron en evidencia la tensión respecto de los valores que debían regir la práctica del hockey -jugar por diversión o jugar para difundir el hockey-y dieron lugar a distintas formas de organización del juego y de reclutamiento e identificación de los jugadores.

El análisis de las formas y espacios de expresión de dichas cuestiones permite determinar cuatro subperíodos: el primero que abarca de 1903 a 1908, el segundo de 1909 a 1914, el tercero de 1914 a 1919 y por último el transcurrido entre 1919 y 1930. 
Identidades y valores en tensión: los inicios del hockey sobre césped en Argentina

Del juego amistoso y mixto entre residentes británicos radicados en Argentina a los equipos divididos por sexo (1903-1908)

Los comienzos de la práctica del hockey sobre césped en Argentina se remiten al año 1903, cuando un grupo de mujeres y hombres procedentes de Gran Bretaña "entusiasmados por practicarlo" comenzaron a reunirse en equipos mixtos identificados por el lugar de residencia. Quienes lo jugaban eran aquellos y aquellas que lo habían practicado en su país de origen y que deseosos de reproducir su vida en el nuevo país, comenzaron a jugarlo sin otra motivación que el divertirse en los ratos libres. El primer partido que reporta el diario tuvo lugar en septiembre de 1903, en la cancha de Hurlingham, entre dos equipos mixtos conformados con cinco mujeres y seis hombres cada uno; un equipo representó a Buenos Aires y el otro a Venado Tuerto. Sus apellidos permiten afirmar el origen británico de los jugadores y jugadoras.

Probablemente la exhibición del juego de ayer en Hurlingham ha sido la primera en ser vista dentro de los alrededores de Buenos Aires, y por el fervor general demostrado no hay duda que el hockey será incluido en la lista de los deportes y será tomado con fervor como otros. Fue organizado por iniciativa de la Sra. C. Hay y fue jugado en Hurlingham. (The Standard, 18 de septiembre de 1903, p. 8.)

El creciente entusiasmo derivó en que la modalidad de organización de partidos también fuera por sexo y bajo la guía de instituciones. En este sentido, el primer partido oficial de hockey femenino, el primer torneo de hockey de caballeros y los inicios de la conformación de la Asociación Argentina de Hockey (A.A.H.) pueden ser interpretados como evidencias de una práctica asistemática pero de incipiente institucionalización. Escuelas y clubes vieron en el deporte un instrumento para la formación y modelaje del carácter, un medio de aprendizaje para la vida poniendo en juego el respeto a las tradiciones, la generosidad, la disciplina y el control de emociones y/o pasiones.

Las escuelas inglesas fueron el espacio deportivo de las mujeres, de la mano de docentes llegadas de Inglaterra. Las primeras en jugarlo fueron las alumnas de Alexandra College de Belgrano, Quilmes High School y St. Catherine's School de Quilmes ${ }^{4}$. La identificación de apellidos no británicos en la nómina de jugadoras

4 The Standard, 20 de junio de 1908. permite suponer la presencia de hijas de madre inglesa y padre español-criollo en la matrícula de escuelas británicas.

Meses más tarde, a partir de la práctica en las escuelas y con el objetivo de educar a las niñas "en el sentido de justicia y camaradería propia de los ingleses" la Quilmes High School decidió fundar un games club que ofreció regularmente la práctica de hockey, tenis y cricket.

Por otro lado, los caballeros se organizaron en clubes -San Martin Rovers Hockey Club y Buenos Aires en 1905, Belgrano Athletic Club, San Isidro A.C. y Pacific Railway Athletic Club en 1908. Identificados con el lugar de residencia y por la ubicación de las canchas, los jugadores de San Isidro y Belgrano adoptaron el nombre del barrio para denominar a sus equipos.

El crecimiento del hockey y el fervor de sus seguidores hicieron que se propusiera conformar la Liga de Hockey Argentina y que el dueño del Buenos Aires Herald donara una copa de plata, a la que llamó Copa Herald. La misma debía ser disputada en un torneo interclubes para el que era condición la participación de cuatro equipos como mínimo y ser al menos tres los clubes representados. Los clubes que participaron fueron Belgrano A.C. (dos equipos), San Isidro y Pacific Railway Club. La copa quedó en posesión y bajo la dirección del comité de hockey del Belgrano Athletic Club hasta que la Asociación estuviera definitivamente constituida.

A mediados de 1908 The Standard se convirtió en vocero de aquellos que se decían interesados por comenzar a jugar al hockey seriamente en el país:

Desde hace tiempo se habla entre los entusiastas del juego de comenzar a jugar hockey seriamente en este país. Este proyecto está a punto de cristalizarse, tanto es así que ya se han jugado algunos torneos eliminatorios entre unos pocos aficionados. Se propone ahora jugar regularmente partidos en la cancha de Belgrano A.C. Seguramente habrá muchos hombres llegados del "viejo terruño", quienes habiendo jugado en casa quieran continuar la práctica de éste, uno de los mejores juegos. Cualquier jugador que quiera jugar regularmente puede enviar su nombre, dirección y posición en la cual juega a G. Morton, Sucre 1821, Belgrano o a H. Brookhouse, Bartolomé Mitre 544, Buenos Aires. (The Standard, 1 de julio de 1908, p. 8)

5 The Standard, 29 de septiembre de 1908, p 13. 
¿Qué significaba el calificativo "seriamente"? ¿Acaso jugar en equipos mixtos, por amor al deporte o por el placer en el juego mismo, de hecho coincidente con los valores fundantes de los deportes y pasatiempos ingleses, no era jugar seriamente? ¿0 la calificación de "seriamente" tenía que ver con la idea de jugar separados por sexo y representando instituciones? Tal vez la respuesta a estas preguntas deba buscarse en las características que iba adquiriendo el deporte moderno, en términos de jugar en búsqueda del resultado para el mejor posicionamiento de ese equipo dentro de la estructura social deportiva. Probablemente la creación de la Asociación Argentina de Hockey responda a este encuadre. En agosto de 1908 The Standard informó a la comunidad que la primera comisión directiva había sido constituida. Los apellidos de dirigentes elegidos no dejan duda de su pertenencia a la comunidad británica: presidente: Sr. Thomas Bell; vicepresidente 1ro: Sr. G.E. Morton (Belgrano); vicepresidente 2do: F.R. Gruppy (San Isidro); secretario: Sr. H. Brookhouse (Belgrano); tesorero: Sr. B. Daine (Pacific); vocales: F.W. Sewards (San Isidro), R.J. Nicoll (Belgrano) y H.G. Stone (Pacific).

De allí en más el juego del hockey ya no sería solo una cuestión de vecindarios ni de clubes que jugaban por placer como espacio de esparcimiento; además, las escuelas también lo incorporaron como actividad educativa y los clubes ingleses, como espacio exclusivo para los caballeros, lo incluyeron en su lista de actividades. Se podría afirmar que la formalización de la A.A.H., como punto de inflexión en la manera de institucionalizar la práctica y en los criterios para la conformación de los equipos, marca el inicio del segundo período.

\section{Hacia una práctica seria (1909-1914)}

El segundo período estuvo caracterizado, según el diario, por la seriedad con que era encarado el juego, por la relevancia de los valores del deporte amateur, más la tendencia a la formalización e institucionalización creciente de su práctica.

Como resultado del crecimiento del hockey en la etapa anterior se decidió solicitar la afiliación de los clubes a la Hockey Association of England (HAE) ${ }^{6}$. El diario remarcaba que el hockey en Inglaterra nunca había sido

6 La Hockey Association of England, fundada en 1875, era la organización reguladora de la práctica de este deporte en el mundo. jugado por una copa o trofeo, exceptuando la Army Cup y Hospital Cup ambas con fines caritativos ${ }^{7}$. Ese dato parece haber sido determinante para que la A.A.H. dispusiera que el hockey en Argentina debía ser jugado bajo los mismos criterios de la Hockey Association of England. La práctica deportiva se mantuvo en el marco del amateurismo aceptado, aparentemente, sin discusión. Todo cuestionamiento al respecto no era aceptado y la Copa Herald, donada con el propósito de que fuera disputada cada año, no fue incluida en el programa de la A.A.H. En consecuencia, la copa jugada el año 1908 quedó para San Isidro como ganador definitivo (The Standard, 16 de abril de 1909).

La valoración dada a la práctica del hockey, incentivada fuertemente por el diario, pasaba fundamentalmente por el espíritu bajo el cual debía ser jugado. Jugar por amor al deporte significaba, en palabras de The Standard, hacerlo con generosidad, sin egoísmo y para el éxito del equipo sin considerar premios o posiciones. Las derrotas no debían ser consideradas de poca, sino de nula importancia.

Una de las mayores ventajas es su absoluto amateurismo, siempre ha sido jugado solamente por amor al deporte. Los dirigentes de la Asociación de Hockey Inglesa advirtieron estrictamente acerca de jugar por copas y todo tipo de trofeos. (The Standard, Hockey,19 de abril de 1909, p. 9)

El diario señalaba permanentemente, luego de cada encuentro, que los jugadores demostraban que la práctica del hockey se llevaba a cabo en un ambiente de camaradería y diversión donde el único beneficio para el equipo era jugar. Para el diario no había duda de que la práctica del hockey era un espacio de transmisión y reproducción del espíritu inglés: jugar con generosidad y por amor al deporte.

Los clubes también utilizaron las páginas del diario para fijar posiciones y hacer las convocatorias para formar sus equipos; Belgrano A.C. resaltaba que una de las ventajas del juego era el absoluto amateurismo y que siempre debía ser jugado por amor al deporte ${ }^{8}$. Lo mismo hizo Lomas Athletic ${ }^{9}$.

La cantidad de equipos inscritos en la A.A.H. y presentes en las canchas (Belgrano A.C., San Isidro, Pacific Railway Athletic Club, Buenos Ayres Great Southern

7 The Standard, 16 de abril de 1909.

8 The Standard, 19 de abril de 1909.

9 The Standard, 6 de mayo de 1909. 
(B.A.G.S), Lomas Athletic Club y Buenos Ayres Hockey Club) mostraba que los valores dispuestos por la H.A.E. y adoptados por la A.A.H. parecían estar fuera de toda discusión. Y esta actitud queda ilustrada en artículos que afirmaban "el juego que crece (...) el hockey vino a quedarse". O como significó para un jugador de Belgrano A.C.:

(...) este deporte está obteniendo cada vez más apoyo en esta parte del país, no pasarán muchos años antes de que este importante y científico pasatiempo ocupe un espacio igual al de sus deportes rivales. (The Standard, Belgrano A.C., 18 de abril de 1910, p. 2)

Pero no todo se produjo en un clima de coincidencias. El reconocimiento de la A.A.H. por parte de la H.A.E. en iguales condiciones que las demás asociaciones de hockey extranjeras, reavivó la tensión entre jugar por copa o jugar en las líneas del juego amateur. En ese marco, con una clara actitud intervencionista y en respuesta a grafitis y cartas enviadas a favor de competir por copa, el editor de The Standard expresó:

Este es un juego para amateurs y solo amateurs, sabiendo cómo la promoción de competencias de liga por copa han influenciado en la declinación del fútbol amateur, la English Association prudentemente ha fijado su postura en contra de promover una copa o competencias de liga de cualquier característica. No hay duda de que la copa es simplemente la cuña principal para el profesionalismo. Sinceramente espero que este juego continúe siendo jugado en lo saludable del amateurismo. Vale la pena seguir jugando solamente por amor al juego. (The Standard, Hockey chat, 24 de julio de 1910, p. 5)

De esta forma el diario, sin explicitar sus fundamen$\operatorname{tos}^{10}$, puso en escena el tema del profesionalismo condenando toda forma de competencia por considerarla el paso previo a la pérdida del amateurismo. Se enfrentaban así dos formas de concebir su práctica: el hockey como pasatiempo y el hockey por torneos y copa. La primera postura, sostenida por la A.A.H. y el diario, determinaba que la formación de equipos y la organización de encuentros debían ser puramente de carácter amistoso. Por otro lado, quienes aspiraban al juego por copa o trofeo lo hacían con la convicción de que la copa generaría mayor interés. Y por ello debía ser conside-

10 Cabe señalar que el fútbol en la Argentina recién fue formalmente profesionalizado a principios de 1930 (Frydemberg, 1999). rado un medio legítimo para estimular el crecimiento del hockey en la Argentina.

Estas diferentes maneras de concebir el juego suponían posicionamientos encontrados en relación a los valores que debían guiar la práctica del deporte y a los objetivos que pretendían alcanzarse a través del juego. Quienes enfatizaban el concepto de pasatiempo, vinculaban los valores del juego amateur con el intento de mantener la identidad del país de origen, o la falta de deseo de integrarse a una nación en la que los "ingleses" se sentían de paso. De allí que sostener el juego amateur podría ser tomado como un rasgo identitario: el hockey pasatiempo de británicos y entre británicos. Por otro lado, quienes tenían interés en las competencias por copa se posicionaban como actores imbuidos de los valores del deporte moderno y cosmopolita, movidos por el deseo de difundir el hockey en esta parte del mundo y más allá de ellos mismos. La postura del diario aparece como una tercera posición. Para The Standard los valores del deporte amateur (no copa, no trofeo) no se ponían en duda. Sin embargo, lo que sí le interesaba al diario era el crecimiento del hockey más allá de la comunidad británica. En reiteradas ocasiones se pudo leer su inquietud por la no participación de los argentinos: “(...) Nos preguntamos por qué los argentinos no han comenzado con este deporte, es rápido, excitante y no hay reglas complicadas" (The Standard, 5 de octubre de 1913)

En síntesis, tres posturas convivían: el hockey como pasatiempo entre ingleses, el hockey por copa para generar mayor participación y el hockey basado en los valores del deporte amateur pero con interés en la difusión más allá de la comunidad británica, quizás con la expectativa de incrementar el número de lectores del diario.

Durante los años 1911 y 1912 este panorama tuvo algunos cambios. En primer lugar, cuando según el diario todo transcurría normalmente y con buenos augurios para la temporada, los caballeros evidenciaron dificultades para lograr conformar los equipos; es decir, era recurrente que los equipos no pudieran completar el número de jugadores reglamentarios. En segundo lugar, los equipos presentes en las canchas se prestaban jugadores para completar sus planteles o se intercambiaban jugadores en pos de un juego técnico-táctico más equilibrado.

Tales actitudes derivaron en cierta tensión entre entusiastas y el diario acerca de qué era moralmente correcto. The Standard entendía que la no asisten- 
cia de los jugadores a la cancha era signo de falta de compromiso, y que no dar todo en la cancha a pesar de las diferencias iba en contra del verdadero espíritu del deporte. Como vocero de lo que ocurría en las canchas, y como acérrimo opositor al profesionalismo y al intercambio de jugadores, puso en discusión la realidad que se atravesaba y la repercusión que tendría sobre del crecimiento y los valores del hockey. The Standard, como "mesa de debate", reflejó los diferentes enfoques respecto a la causa por la que "el amor por el juego" se había visto afectado. En opinión de un lector, la razón del desinterés por asistir a los juegos estaba en la falta de campeonatos; una vez más entraban en escena los términos torneo y trofeo. The Standard sostenía que la práctica de intercambiar jugadores entre clubes, durante el partido, era la causante de la ausencia de jugadores en los planteles:

No hay copa, no hay liga, solo hay fixture. Es difícil para los equipos que se presenten completos pero los miembros de los clubes deben comprender que una promesa es una promesa. (The Standard, 06 de agosto de 1912, p. 4)

Para quienes defendían el juego por una copa, el intercambio de jugadores iba en detrimento del interés en el armado de equipos estables que se midieran a lo largo de un campeonato para definir un ganador. Pero a juzgar por la actitud de algunos equipos -que se presentaban a los partidos con menos jugadores de los reglamentarios y completaban sus planteles con miembros del equipo contrario para hacer posible el juego, o para lograr un juego más equilibrado sin importar la camiseta-, la prioridad era el interés por sostener un juego entretenido y no el deseo de ganar por encima de la calidad del buen juego. Muchas de las notas dejaban ver que el espíritu de diversión y caballerosidad regía el juego por sobre todas las cosas. El interés estaba en jugar más allá del resultado para el club. La polémica postura del diario The Standard -que sostenía que jugar por copa era un paso hacia el profesionalismo, y que el intercambio de jugadores era la causa de las ausencias y por ello iba en detrimento del hockey- podría ser entendida en el marco de las razones de una empresa comercial: el crecimiento del hockey en la Argentina bajo los lineamientos del deporte amateur y más allá de la comunidad británica, podría derivar en un incremento de número de lectores; y el mismo efecto podría acontecer si alguna cuestión polémica era planteada a través de las páginas del diario. ¿Por qué no iban a las canchas? ¿Era por la falta de torneo por copa? ¿Guardaba alguna relación con los cronogramas de partidos de modo que se superponían con los de los partidos de otros deportes?

En este punto resulta importante recordar que para los británicos ser sportsmen implicaba realizar diferentes deportes y no la búsqueda primaria de ser brillante en uno determinado. Además, el espacio de las mujeres también se había visto afectado. El ímpetu demostrado con la práctica en las escuelas y la inclusión del hockey femenino en los clubes, que en algún momento hicieron pensar en un futuro promisorio, parecía haberse evaporado. Deseoso de que la ausencia de las chicas fuera temporaria, The Standard expresaba su esperanza de que volvieran a la cancha como en los tiempos cuando "Quilmes hizo rodar la bocha y Belgrano lo siguió".

La estructura social de la vida moderna, con el trazado de ferrocarriles y empresas, hizo que los ingleses se radicaran tanto en la ciudad como en el campo. Orientados a fortalecer la vida de los británicos en Argentina, y posiblemente tomando la costumbre de la gente del cricket, la A.A.H. decidió organizar el partido Town vs. Camp para el hockey. El diario utilizó sus páginas para invitar a los residentes del campo interesados en jugar al hockey. El primer encuentro de Town vs. Camp fue programado para junio de 1911 en la cancha de Belgrano A.C. Y cada temporada se completaba con partidos amistosos, partidos representativos ${ }^{11}$ : Town and Camp, Cities and Suburbs y North vs. South.

En medio de cuestionamientos a la A.A.H. por no hacer esfuerzos para estimular el hockey y organizar partidos de exhibición en Córdoba, Rosario o Montevideo, se declara la guerra entre Inglaterra y Alemania. Otra historia comenzaba a trazarse. Los años coincidentes con el desarrollo de la Primera Guerra Mundial son la etapa que aborda este tercer subperíodo.

11 Los partidos representativos eran aquellos en los cuales cada uno de los equipos estaba conformado por una selección de jugadores que podían pertenecer a un club, un vecindario, una nacionalidad o una empresa determinada, cuya identidad representaban en el encuentro. 


\section{La práctica del hockey se detiene (1915-1919)}

El devenir de la guerra en 1914 fue un punto de inflexión a partir del cual cambió la práctica del hockey en Argentina. Los esfuerzos por continuar el deporte a pesar de la guerra no prosperaron. Durante casi cinco años la comunidad británica estuvo concentrada en lo que ocurría en sus tierras y ausente de las canchas de hockey. La guerra repercutió en la vida de los ingleses radicados en Argentina. A pesar de la buena posición en que se encontraban, los británicos no dudaron en alistarse para servir en la Gran Guerra. "Muchos han sacrificado un buen y confortable estilo de vida". La principal preocupación de los hombres era ofrecerse como voluntarios para servir a la "gran causa". Entre algunos de los voluntarios que partieron se encontraban jugadores de los principales clubes (San Isidro, Belgrano A.C., Quilmes A.C.). Las mujeres, organizadas en la Asociación Patriótica de Mujeres Británicas, se abocaron a recaudar fondos para la Cruz Roja y las familias de los soldados. La finalización de la guerra marca el inicio de una etapa que estuvo marcada por el resurgimiento y crecimiento de la práctica del hockey en la Argentina

\section{El hockey como práctica deportiva moderna y por fuera de la comunidad británica (1919-1930)}

En julio de 1919, The Standard retomó la publicación de avisos referidos al hockey y su interés por la difusión del deporte. En ellos el diario dejaba nuevamente planteada la polaridad entre el jugar por placer y el jugar por copa o trofeo, aún en medio de la incertidumbre respecto al renacimiento del hockey y del lento reintegro de aquellos jugadores que habían vuelto de la guerra.

La participación de otros deportes en torneos por trofeos alertó a la A.A.H. y al diario The Standard acerca de la necesidad de un cambio para revivir el hockey en Argentina. Con una mirada contraria a los períodos anteriores, la A.A.H. y el diario avalaron la idea de establecer el campeonato por copa. El cambio en la modalidad de organización era un hecho.

El restablecimiento del campeonato de la A.A.H. para los caballeros y la presencia de cinco clubes y ocho equipos, hicieron pensar en un futuro promisorio que no condijo con la realidad. A partir de 1924 el hockey masculino disminuyó considerablemente. Si bien la cantidad de clubes representados en la liga durante 1924, 1925 y 1926 se mantuvo como hasta 1923, no fue lo mismo con la cantidad de equipos. Solo cinco equipos integraron la liga de caballeros.
Una vez más se estancaba el hockey de caballeros. A criterio de la A.A.H. y del diario dos cuestiones limitaban el crecimiento del hockey. En primer término, la escasa infraestructura existente. La falta de canchas generaba muchas dificultades al momento de organizar las prácticas y/o partidos de los diferentes deportes, entre ellos el hockey. La A.A.H. lo expresó en las páginas del diario: “(...) Aún la escasez de canchas retarda el desarrollo natural de este deporte. Este es un problema que lamentablemente afecta a muchos clubes" (The Standard, 13 de abril de 1928. p. 7).

En segundo término, la cantidad de hombres disponibles en las canchas no era suficiente para jugar al hockey. El escaso ingreso de jugadores nuevos y jóvenes al mundo del hockey y la superposición de programas deportivos hacía muy difícil para los clubes la formación de equipos. A juzgar por las noticias del diario, el interés de los jugadores hacia otros deportes, principalmente el fútbol, afectaba al hockey. En reiteradas oportunidades se debieron reprogramar partidos porque los jugadores se encontraban jugando al fútbol. Ilustra tal conjetura la publicación de Pacific R.A.C.:

Nuevamente es de lamentar que la convocatoria de los domingos para jugar al fútbol sea de preferencia para los hombres que hacen deporte; por esto los partidos de hockey programados para ser jugados por la tarde debieron ser jugados por la mañana para desilusión de muchos de los seguidores. (The Standard, Notes and Comments, A.A.H., 13 de abril de 1928, p. 7)

A ello se debió la reprogramación del partido entre San Isidro y B.A.H.C del 29 de mayo de 1925. La cancha estaba ocupada con un partido de fútbol. También los partidos de fútbol se jugaban los días domingos por la tarde, el mismo día designado para el hockey de caballeros. Esto hizo que posteriormente se dispusiera de otro día para los partidos de hockey.

De la lectura de The Standard puede advertirse claramente que el restablecimiento del hockey de caballeros en Argentina fue un proceso con aceptación continua pero de crecimiento irregular. Sin lugar a dudas se podría afirmar que a partir de 1928, y luego de superar diferentes dificultades, la incorporación del deporte entre los hombres comenzó un proceso de afianzamiento destacable. Se llegó a contar con siete clubes afiliados, nueve equipos y un promedio de 70 partidos del campeonato de liga y nueve partidos por la copa Knock Out. 
Para el caso del hockey femenino, los encuentros que habían sido organizados en carácter de amistosos desde 1908 hasta 1923 pasaron a estar regidos por la Liga de Hockey Femenino, formada en junio de 1924. Los datos atestiguan la existencia de un florecimiento de instituciones que ofrecieron el hockey para las mujeres. A los tradicionales equipos de escuelas se les sumaron equipos formados a partir de la iniciativa de exalumnas de las escuelas. El crecimiento del hockey femenino fue de tal notoriedad, que iniciado el año 1928 se concretó el deseo expresado por el editor del diario en el año 1925 de armar dos divisiones. Cada temporada denotaba mayor aceptación. El sentimiento de pertenencia generado por el hockey se vio reflejado en otras actividades sociales que realizaron identificándose por el hockey. Una de ellas fue el coro al que llamaron Hockeysists are we. La primera presentación fue minutos antes del inicio de un baile que se llevó a cabo el 17 de julio de 1929.

A partir de 1924, con la formación de la Liga, la práctica del hockey femenino no dejó de crecer. Temporada tras temporada hubo indicios de progreso y estabilización. La práctica que había comenzado con un puñado de damas de origen británico fue ganando terreno hasta llegar a formar dos divisiones con un total de catorce equipos. Cabe mencionar que en algunos de los equipos es posible identificar apellidos no británicos y se llegaron a jugar 98 partidos en el año 1928, 117 en 1929 y 123 partidos en 1930. El campeonato de la liga de caballeros, la liga femenina, la copa Knock out y los representativos North vs. South y Argentinos vs. Británicos eran las actividades centrales de cada temporada.

Si bien la comunidad británica concebía el hockey como una práctica de ellos y para ellos, hay publicaciones en The Standard que permiten abrir un marco de posibilidad acerca de la difusión del juego más allá de los británicos. Una de las situaciones en las que esto quedó evidenciado fue en el año 1927 cuando la comunidad alemana, con intenciones de estimular la práctica del deporte entre los suyos, solicitó la colaboración de la A.A.H. para organizar un partido de exhibición. El partido se llevó a cabo el 9 de octubre de ese año; se jugó un partido entre un equipo británico y un equipo de Belgrano, llamados "los alemanes". Los apellidos de la lista de juradores publicados en el diario dan cuenta de las respectivas nacionalidades ${ }^{12}$. Paulatinamente se daría la participación de jugadores de otras nacionali-

12 The Standard, $\overline{9 \text { de octubre de } 1927 .}$ dades y una incipiente apertura de la práctica del hockey más allá de la comunidad británica:

En el día de ayer la comunidad alemana celebró un día de fiesta en la cancha de fútbol de Sportivo Barracas Club. En conexión con la A.A.H. fue invitado un equipo de jugadores británicos locales para jugar con el equipo de Belgrano H.C., equipo alemán que ha hecho las cosas muy bien durante la temporada. La A.A.H., para complementar el equipo alemán, formó un equipo fuerte. Ganaron británicos por 5 a 1 . Mace fue el único jugador que no es británico pero de antemano se había especificado que no iba a ser posible tener un equipo solo de británicos, que seguramente se debería completar con uno o dos argentinos, como ha pasado. Árbitros. Sres. Ziss y R.W. Holdsworth. (The Standard, 10 de octubre de 1927)

Un año después otra noticia sorprendía al mundo del hockey. Al interés por el deporte vivido en el medio local se le debió sumar la aceptación que tuvo en el interior del país. La primera noticia fue desde Rosario, cuando la columna Rosario Letters del diario The Standard del 8 de septiembre de 1928 informó que dos equipos rosarinos de caballeros habían jugado un partido en Plaza Jewell. De ahí en adelante se los pudo ver con asiduidad e incluso viajaron a Buenos Aires para jugar con equipos locales como Quilmes "A", Pacific R.A.C., B.A.G.S. y B.A.H.C.

El año 1930 fue testigo de mayores indicios que la práctica del hockey se afianzaba en el interior y que algunos argentinos se animaban a jugarlo. Equipos como el de Junín (que formó con nueve jugadores de apellido británico y dos de apellido no británico) merecieron que el editor de The Standard expresara lo agradable de ver a argentinos tomando parte del juego. La solicitud de la A.A.H. para que se incluyera la nacionalidad de jugadores y jugadoras en la lista, de buena fe abona la hipótesis de que el hockey comenzaba a ser jugado por no británicos.

De todos modos no debe confundirse este interés por ampliar las redes de sociabilidad a través del hockey hacia otros grupos no ingleses con el deseo de asimilarse o de perder sus rasgos identitarios de británicos en la Argentina: "Somos británicos, y seguiremos siendo británicos, pero en este país hay un lugar para todos los hombres de bien y legítimo interés" (The Standard, 10 de agosto de 1916), expresaron en ocasión del centenario de la independencia. Continuamente había indicadores que afirmaban esta postura. La organización 
de giras deportivas con los ingleses radicados en países vecinos como Chile y la República Oriental del Uruguay era otro ejemplo, en tanto reafirmaba una vez más el sentido de comunidad de los "ingleses" y sus descendientes.

Si bien la situación nacional e internacional producida en 1930 produjo una nueva transformación de la sociedad, en donde se desdibujaron aún más los límites entre habitantes de una comunidad u otra, esto no se vio reflejado en la comunidad inglesa. El ser inglés y vivir como inglés en la Argentina estaba por sobre toda situación. Como ha podido verse, el análisis del modo en que la práctica del hockey fue paulatinamente incorporada a la rutina de sus vidas permite dar cuenta de la relevancia de la práctica deportiva dentro de su cultura. Los inmigrantes procedentes de Gran Bretaña continuaban arribando a la Argentina para trabajar, en su mayoría en compañías británicas, y el diario era el medio por el cual eran invitados a incorporarse a los clubes.

El espíritu de no integración también quedó de manifiesto ante comportamientos vistos en la cancha. En 1929, la discusión entre un jugador y el árbitro fue señalada como una actitud no acorde a los valores en los que debía identificarse a un inglés. Quien usara las páginas del diario para expresar su desaprobación calificó el proceder como una "característica del fútbol latino", no acorde al espíritu amateur (The Standard, 23 de septiembre de 1929).

Como se ha podido leer, la relación entre la práctica y los valores del hockey sobre césped en Argentina estuvo atravesada por acuerdos y diferencias en torno a la organización del hockey como rasgo identitario de la comunidad británica. El espíritu del deporte amateur y la adopción del hockey como práctica se desarrollaron en un transcurrir de diferentes maneras de concebirla, y las canchas fueron el reflejo de lo que ocurría. Lo cierto es que a pesar de los cuestionamientos del diario y de algunos entusiastas, los jugadores continuaban con la actitud de jugarlo entre los británicos y como aspecto para seguir "viviendo a la inglesa". Los valores que sustentaban la práctica del hockey -amor por el deporte, jugar a pesar de las diferencias- eran sostenidos por sobre toda dificultad y todo cuestionamiento. Tal vez se pueda imaginar que los británicos no tuvieron otra intención más allá que la de relacionarse entre ellos y que el hockey era un medio más para el fortalecimiento de su identidad en un país ajeno. Si bien el diario, como actor de relevancia y con el propósito de aumentar los lectores problematizó diferentes cuestiones, también puso a discusión las reglas sobre las cuales debía construirse la práctica. Lo hizo, finalmente, convirtiéndose en un medio de difusión eficiente para los interesados en el deporte más allá de la comunidad británica.

\section{Referencias}

Archetti, E. (2001). El potrero, la pista y el ring. Buenos Aires: Fondo de Cultura Económica.

Devoto, F. (2003). Historia de la inmigración en la Argentina. Buenos Aires: Editorial Sudamericana.

Elias, N. y Dunning, E. (1986). Deporte y ocio en el proceso de la civilización. Madrid: FCE.

Elias, N. y Dunning, E. (1992). Deporte y ocio en el proceso de la civilización. Buenos Aires: Fondo de Cultura Económica.

Frydemberg, J. (1999). El nacimiento del fútbol profesional argentino: resultado inesperado de una huelga de jugadores. Revista digital www.efdeportes.com. Año 4, № 17. Buenos Aires.

Graham-Yooll, A. (2007). La colonia olvidada. Tres siglos de habla inglesa en la Argentina. Buenos Aires: Emecé Editores.

Guttman, A. (1994). Games and empires. New York: Columbia University Press.

Mandell, R. (1984). Sport. A cultural History. New York: Columbia University Press. 